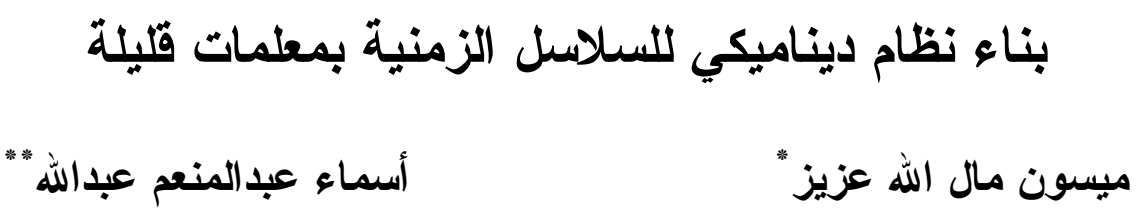

الملخص

في هذا البحث نم استخدام طريقة دالة القاعدة النصف قطرية (الثـــعاعية)

كلبناء نماذج لسلسلة زمنية ، إذ نم استتناج نموذج لسلسلة زمنيـــة (radial basis) غير خطية وتبين ان الخطأ محصور بين الصفر و الو احد ووضـــت الخصــائص الاحتمالية لهذه السلسلة مع تحليل كامل لبو اقي النموذج ومحاكاة بيانــات النمــوذج وفحصها إذا كانت البيانات المولدة من النموذج لها خصائص البيانات الخام نفسها ـ

\title{
Building Parsimonious Dynamic System for Time Series
}

\section{ABSTRACT}

In this paper the radial basis function method is used for the generation of models of time series. It has been arrived at a model for nonlinear time series. It has also been found that the error is restricted between zero and one. The probability characteristics have been explained for such series accompanied by a complete analysis for the residuals of model. A simulation has been done for the purpose of examination the generative information of the model. which have the same information characteristics.

$$
\begin{aligned}
& \text { " مدرس/ كلية علوم الحاسبات و الرياضيات/جامعة الموصل } \\
& \text { " مدرس مساعد/كلية علوم الحاسبات و الرياضيات / قسم الرياضيات / لريات جامعة الموصل }
\end{aligned}
$$


من المألوف في عملية البناء الديناميكي هو أيجاد دالة من بيانات السلســلة الزمنية إذ أن النظام الديناميكي غالبا ما يكــون خوارزميــا (Algorithmically)

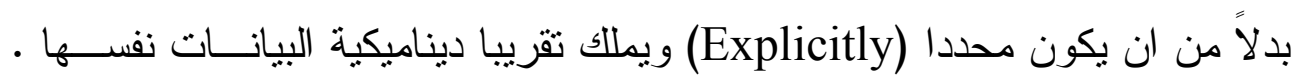
النظام الناتج ويستعمل لـ التخمين (Estimation) ، النوليد (Interpolation) ، السيطرة (Control) ، و لاختبار وجود اللاخطية (Nonlinearity) • ازداد البناء و اصبح اكثر شيوعا كطريقة لفهم موضوع السلاسل الزمنيـــة التي يعتقد انها ممكن ان تحتوي على ميز ات ديناميكية غير خطية ، فضـــا عـنـ Grassberger, 1983; Judd, ) سهولة حساب الاحصائيات كالبعــ الكسـوري 1992) •و على وجه الخصوص بمجمو عات بيانات صغيرة ـ وقد اقترحــ عـدة Farmer \& Sidorowich, 1987; Casdagli, 1989; Mees, ) طر ائق للبناء (1990, 1991; Weigend et al., 1990 الاحصائيين بنماذج السلاسل الزمنية الخطية و اللاخطية وهناك جه قليل في طر ائق

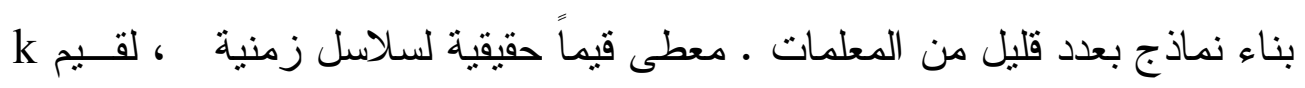
$\left\{Z_{t}\right\},\left\{Y_{t}\right\}$ من المتجهات إذ يفترض (k- Vector valued time series) $Y_{t}=f\left(Z_{t}\right)+V_{t}$

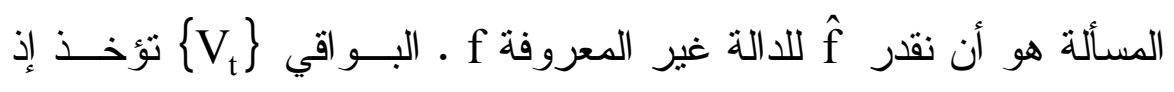

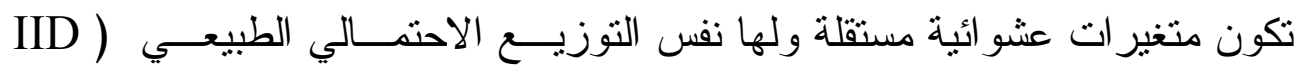


(Independently identically normally distributed) أي (Normal صفر وتباين غير معلوم.

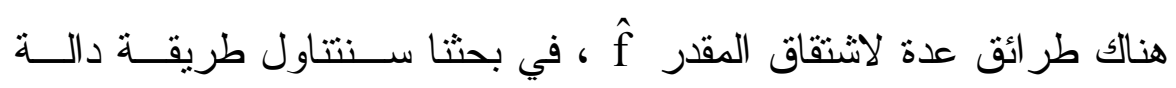

(Radial basis function approach) (القاعدة النصف قطرية (الثعاعية) الجاتب النظري

1. نمذجة دالة القاعدة النصف قطريسـة (الثــعاعية) Radial Basis Function

Modeling

معظم النماذج المحددة غير الخطية للسلاسل الزمنية تملك عدداً كبيرًا مـن

المعلمات ويتم ملاعمتها بعدم وجود التشويش ، تم توضيح كيفية نمذجة دالة القاعدة النصف قطرية (ثعاعية) بعدد قليل من المعلمات ، احد مز ايــا طريقــة القاعـــة النصف قطرية (الثعاعية) هو ان النموذج الناتج يمكن ان يوصف بنموذجاً تحليلياً إذ يمكن در استه كأي نظام ديناميكي أفضل من أن يوصــف بمحاكــاة تجريبيــة ،

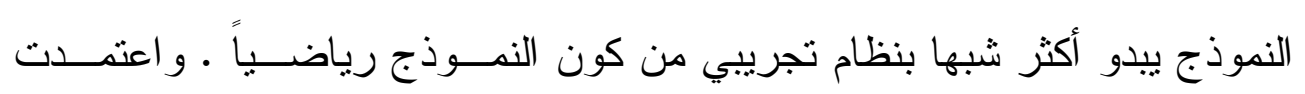
Rissanen, ) (Description Length Criterion) الطريقة على مقياس الطول - $(1989$

2. طريقة Taken's Method :Taken's

نفترض أن yt هي سلسلة زمنية ذات قيم حقيقية وأن (yt واحد

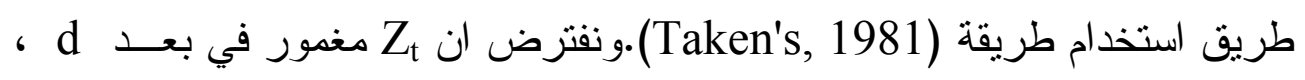
حيث d هو البعد لفضاء الطور الذي فيه الجاذب مغمور ويطلق عليه البعد المغمور 


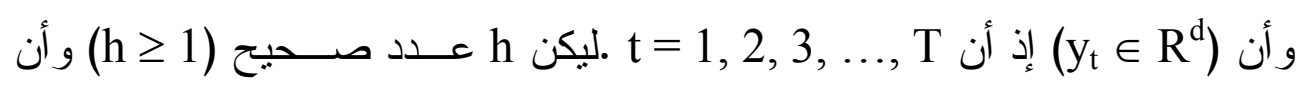
إذاً $\mathrm{T}=\widetilde{\mathrm{T}}-(\mathrm{d}-1) \mathrm{h}$

$\mathrm{Z}_{\mathrm{t}}=\left(\mathrm{y}_{\mathrm{t}}, \mathrm{y}_{\mathrm{t}-\mathrm{h}}, \mathrm{y}_{\mathrm{t}-2 \mathrm{~h}}, \ldots \mathrm{y}_{\mathrm{t}-(\mathrm{d}-1) \mathrm{h}}\right)$

(Mees, 1994)

3ethod of Modeling : طريقة النمذجة

نوجد fo لكي تتلاءم مع السلسلة الحقيقية yt إذ بالإمكان استخدام الندذجـة بطريقة دالة القاعدة النصف قطرية (الثعاعية) (Radial Basis Function) حيث (RB) برمز لها للاختصار

$\hat{\mathrm{f}}(\mathrm{Z})=\sum_{\mathrm{s}=1}^{\mathrm{N}} \mathrm{S}_{\mathrm{s}} \phi\left(\left|\mathrm{Z}-\mathrm{C}_{\mathrm{s}}\right|\right)$

المعادلة (2) هـي نـــوذج القاعـدة النصــف قطريــة (RB) الأصــلية

Basis ( إذ f Original Radial Basis (RB) Model Function و هناك عدة دو ال قاعدة مدكنة في هذا البحث ســوف نأخـــ الدالـــة الكاوسية (Gaussian Function) لأنها تبدو أكثر ملاعمة لنمذجة أنو اع مختلفــة

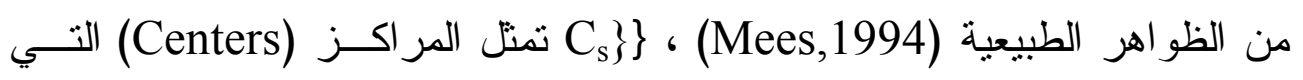

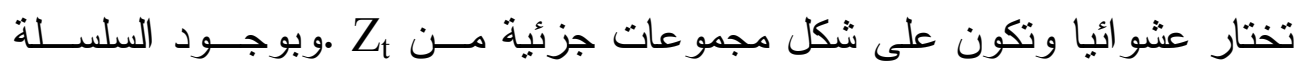
الزمنية Y نقوم بتوليد المتجهات Z نها وذلك باستخدام طريقــة Takens فقــرة

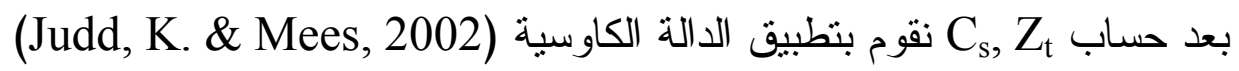

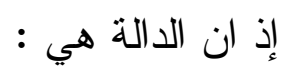
$f(r)=\exp \left(-r^{2} / s^{2}\right)$

$$
\mathrm{r}=\left(Z_{t}-C_{s}\right) \text { عندما }
$$


$\mathrm{f}\left(\left|\mathrm{Z}-\mathrm{C}_{\mathrm{s}}\right|\right)=\exp \left(-\left(\mathrm{Z}-\mathrm{C}_{\mathrm{s}}\right)^{2} / \mathrm{s}^{2}\right)$

إذ s² هو تباين السلسلة الزمنية الحقيقية yt ، من المعادلة (4) سينتج لــدينا

مصفوفة بسعة T*d إذ T تشير إلى طول السلسلة yt ، إذ

$\mathrm{y}_{\mathrm{t}}=\mathrm{y}_{1}, \mathrm{y}_{2}, \ldots, \mathrm{y}_{\mathrm{T}}, \mathrm{t}=1,2, \ldots, \mathrm{T}$

و d هو البعد المغور الذي سوف يتم اختياره ، سيتم حســاب المعلمــة

Sوذللك بنطبيق الخوارزمية الاتية . (Mees, 1993) ، (Mees, 1994)

4. Algorithm : الخوارزمية

$$
\mathrm{m}=0 \quad, \quad \mathrm{Y}=\mathrm{Y}_{\mathrm{o}} \quad, \quad \mathrm{I}^{\mathrm{i}}=\mathrm{I}_{\mathrm{o}}^{\mathrm{i}}
$$

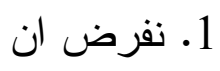

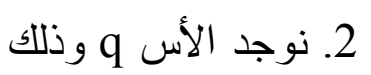

$$
\begin{aligned}
& \mathrm{I}_{\mathrm{m}}^{\mathrm{q}}=\mathrm{Y} . \mathrm{I}_{\mathrm{m}}^{\mathrm{i}} / \mathrm{I}_{\mathrm{m}}^{\mathrm{i}} \cdot \mathrm{I}_{\mathrm{m}}^{\mathrm{i}} \quad \text { over } \mathrm{i} \\
& \mathrm{H}_{\mathrm{m}}=\mathrm{Y} . \mathrm{I}_{\mathrm{m}}^{\mathrm{q}} /\left|\mathrm{I}_{\mathrm{m}}^{\mathrm{q}}\right| \\
& \mathrm{Y} . \mathrm{Y}-\sum_{\mathrm{j}=1}^{\mathrm{m}} \mathrm{H}_{\mathrm{j}}^{2} \leq \theta \mathrm{Y} . \mathrm{Y}
\end{aligned}
$$

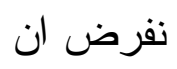

اذهب إلى الخطوة (6) - (6)

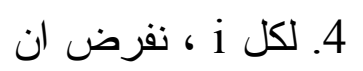

$\mathrm{I}_{\mathrm{m}+1}^{\mathrm{i}}=\mathrm{I}_{\mathrm{m}}^{\mathrm{i}}-\mathrm{I}_{\mathrm{m}}^{\mathrm{i}} \cdot \mathrm{I}_{\mathrm{m}}^{\mathrm{q}} / \mathrm{I}_{\mathrm{m}}^{\mathrm{q}} \cdot \mathrm{I}_{\mathrm{m}}^{\mathrm{q}}$

5. عرف

6. احسب S بو اسطة الحل بالمربعات الصغرى

$$
\text { I(m) S = Y المعادلة }
$$

عندما

$$
I(m)=\left\{I^{i_{1}}, I^{i_{2}}, \ldots, I^{i_{m}}\right\}
$$


The Explanation of Algorithm : شرح الخوارزمية بتطبيق الدالة الكاوسية في المعادلة (4) تتتج مصفوفة ، فنختــار عمــوداً

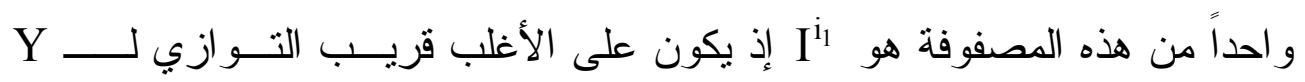
(Mees, 1993) (Most nearly parallel to Y)

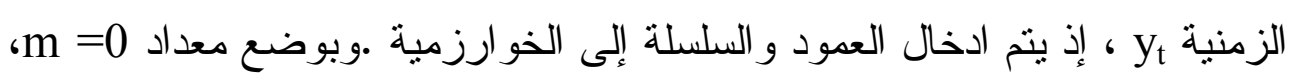

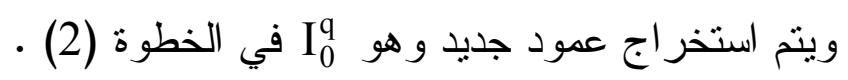
$I_{0}^{\mathrm{q}}=\mathrm{Y} \cdot \mathrm{I}^{\mathrm{i}_{1}} / \mathrm{I}^{\mathrm{i}_{1}} \cdot \mathrm{I}^{\mathrm{i}_{1}}$

ثم يتم حساب : n

$\mathrm{H}_{1}=\mathrm{Y} . \mathrm{I}_{0}^{\mathrm{q}} /\left|\mathrm{I}_{0}^{\mathrm{q}}\right|$ وينم حساب قيمة qu عن طريق

$Y_{t} . Y_{t} \leq \theta Y . Y$

$\Rightarrow \frac{Y_{t} \cdot Y_{t}}{Y . Y} \leq \theta_{t} \quad, t=1,2,3, \ldots, \mathrm{T}$

اذ ان Y بي قيمة من السلسلة الزمنية Y تثم المقارنة كما في الخطوة رقم (3)

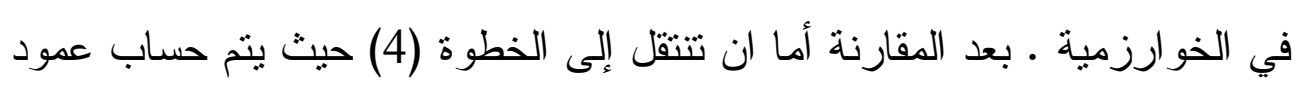
جديد أخر $\mathrm{I}_{1}^{\mathrm{i}}=\mathrm{I}_{0}^{\mathrm{i}}-\mathrm{I}_{0}^{\mathrm{i}} \cdot \mathrm{I}_{0}^{\mathrm{q}} /\left|\mathrm{I}_{0}^{\mathrm{q}}\right|^{2}$

أو نذهب إلى الخطوة رقم (6) بادخال العمود I I يتم حساب المعلمة S باســتخدام طريقة المربعات الصغرى . 6. طريقة المربعات الصغرى: Least Squares Method 


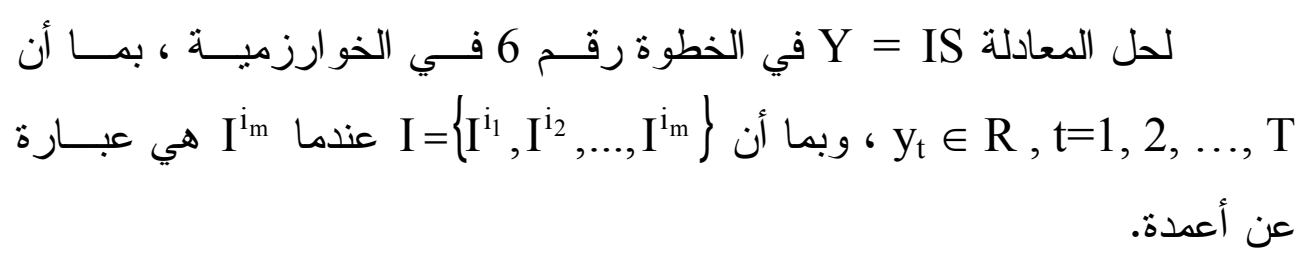

$\mathrm{Y}=\mathrm{IS}+\varepsilon$

$$
\hat{\mathrm{S}}=\left[\hat{\mathrm{S}}_{1}, \hat{\mathrm{S}}_{2}, \ldots, \hat{\mathrm{S}}_{\mathrm{N}}\right] \text { عندما }
$$

$\sum_{i=1}^{N} \varepsilon_{i}^{2}=\varepsilon^{\prime} \varepsilon$

بتعويض المعادلة (5) في المعادلة (6) ينتج

$$
\begin{aligned}
& =(\mathrm{Y}-\mathrm{IS})^{\prime}(\mathrm{Y}-\mathrm{IS}) \\
& =Y Y^{\prime}-2 \hat{S}^{\prime} I^{\prime} Y+\hat{S}^{\prime} I^{\prime} I \hat{S} \\
& \text { وبما أن Scalar) في كمية ثابنت Sch } \\
& \frac{\partial}{\partial \hat{S}}\left(\varepsilon^{\prime} \varepsilon\right)=-2 I^{\prime} Y+2 I^{\prime} I \hat{S} \\
& =0 \\
& {\left[\left(I^{\prime} I\right) \hat{S}=I^{\prime} Y\right] \times\left(I^{\prime} I\right)^{-1}} \\
& \left(I^{\prime} I\right)^{-1}\left(I^{\prime} I\right) \hat{S}=\left(I^{\prime} I\right)^{-1} I^{\prime} Y \\
& \hat{S}=\left(I^{\prime} I\right)^{-1} I^{\prime} Y
\end{aligned}
$$$$
\text { نفاضل (7) بالنسبة الى S ونساويها للصفر }
$$ 
المخطط الاسيابي للخوارزمية

Flowchart of Algorithm

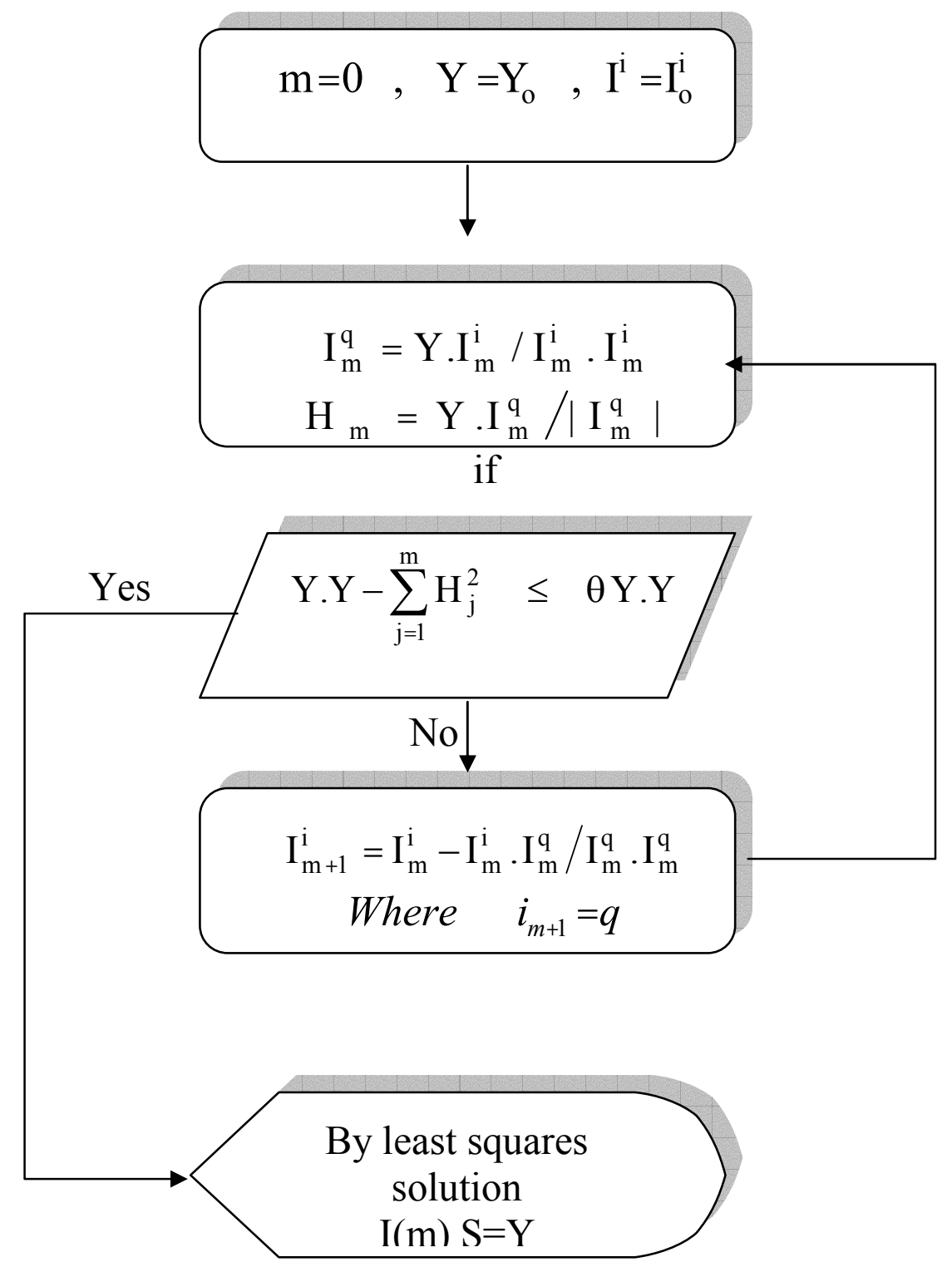




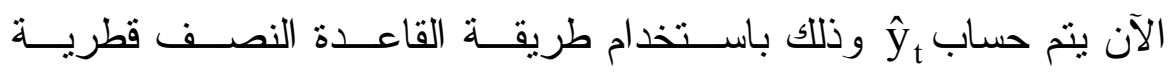

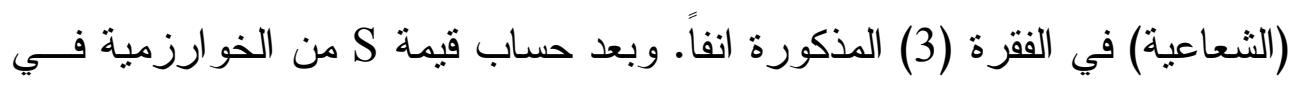
الفقرة (4) السابقة .

$\therefore \hat{\mathrm{y}}_{\mathrm{t}}=\sum_{\mathrm{s}=1}^{\mathrm{N}} \mathrm{S}_{\mathrm{s}} \phi\left(\left|\mathrm{Z}_{\mathrm{t}}-\mathrm{C}_{\mathrm{s}}\right|\right)$

$$
\hat{\mathrm{y}}_{\mathrm{t}}=\hat{\mathrm{f}}\left(\mathrm{Z}_{\mathrm{t}}\right) \text { إذ }
$$

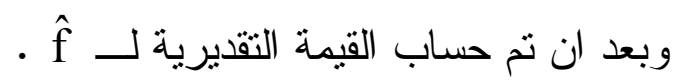

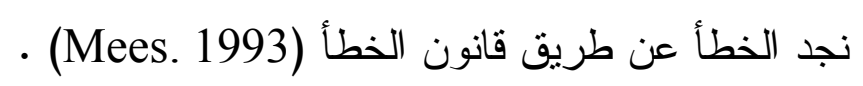
err $=\frac{\sum_{t=1}^{T}\left(\hat{y}_{t}-y_{t}\right)^{2}}{\sum_{t=1}^{T} y_{t}^{2}} \ldots \ldots \ldots .(9)$

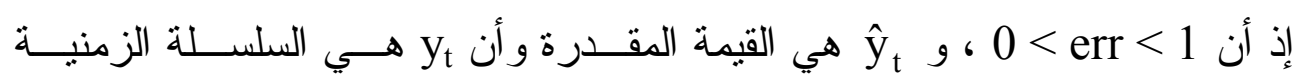
الحققية . ملاحظة :

سوف يتم حساب الــ (err) عند كل اختيار عشوائي للمر اكز إلى ان نصل

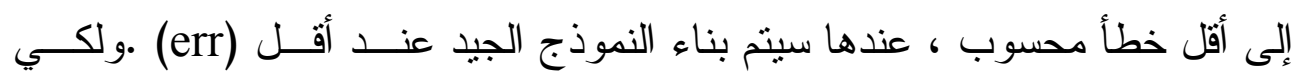

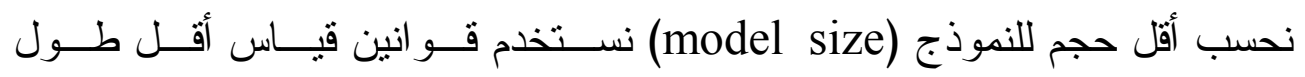
• (Mees. 1993) (Minimum description Length, Rissanen, 1989)

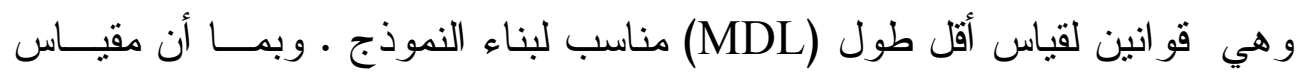

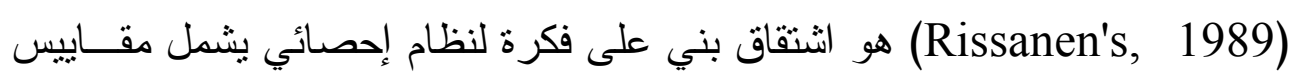
- SIC, BIC : مقياس معلومات أكاكي ومقياس معلومات بيز . . . 7 Akaike's Information Criteria and Bayesian Information Criteria اصبح مقياس معلومات أكاكي أداة قياسية في نمذجــة السلاســل الزمنيـــة

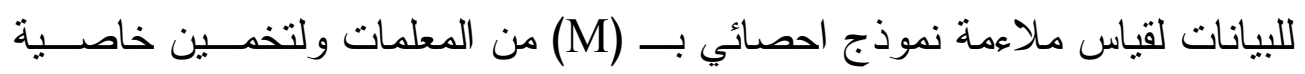
ملاعمة النموذج، قدم Akaike, 1973, 1974a) Akaike مقياس معلومات ، هذا مناس 


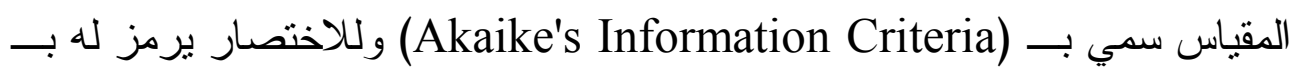
ويعرف بالمعادلة الآتية : (AIC)

AIC $(M)=-2 \ln$ [maximum likelihood] $+2 \mathrm{M}$

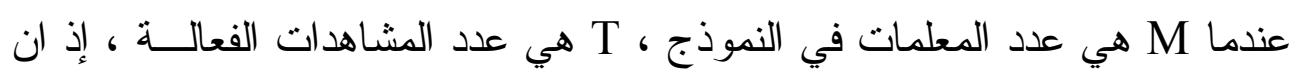
دالة (Log - Likelihood) هي

$L n L=\frac{-\mathrm{T}}{2} \ln 2 \Pi \sigma_{a}^{2}-\frac{1}{2 \sigma_{a}^{2}} S(\phi, \mu, \theta)$

بما ان المعادلة (11) أعظم ما يمكن بالنسبة لــ $\hat{\sigma}_{a}^{2}=\frac{S(\hat{\varphi}, \hat{\mu}, \hat{\theta})}{T}$

$L n L=\frac{-\mathrm{T}}{2} \operatorname{Ln} \hat{\sigma}_{a}^{2}-\frac{T}{2}(1+\operatorname{Ln} 2 \Pi)$

وبما أن الحد الثاني من المعادلة (12) هو ثابت ، اذاً فــان مقيــاس AIC

$\operatorname{AIC}(M)=\mathrm{T} \operatorname{Ln} \hat{\sigma}_{a}^{2}+2 M$

إذ أن الرنبة المثالية للنموذج هو باختبار قيمة M ـ إذ يكون عندها AIC

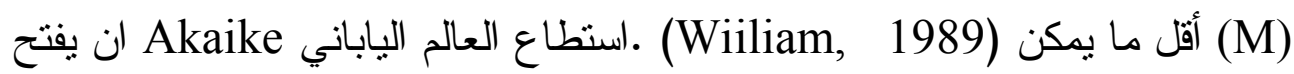

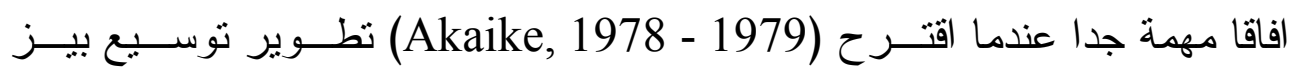

Bayesian Information ) BIC باجر اءات قليلة على AIC (Bayesian)

(Criterion وسماه بمقياس معلومات بيز (BIC) باجر الذي يأخذ الثكل الآتي: $B I C(M)=\mathrm{T} \operatorname{Ln} \hat{\sigma}_{a}^{2}-(T-M) \operatorname{Ln}\left(1-\frac{M}{T}\right)+M \operatorname{Ln} T+\mathrm{M}_{\mathrm{z}} \operatorname{Ln}\left[\left(\frac{\hat{\sigma}_{z}^{2}}{\hat{\sigma}_{a}^{2}}-1\right) / M\right]$

حيث

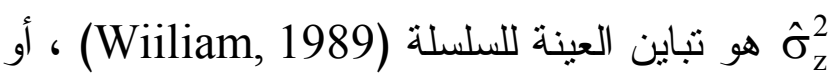

$\mathrm{BIC}(\mathrm{M})=\mathrm{T} \log (\mathrm{err})+\mathrm{M} \log (\mathrm{T})$ 
إذ أن T يمثل حجم العينة المستخدمة ، و M يمثل عدد المعلمــات ، و (err) هــو

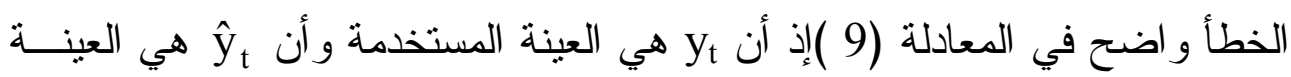
الناتجة (Mees, 1993).

8chwarz Information Criterion :مقياس معلومات شوارز يرمز لمقياس معلومات شــوارز (Schwarz Information Criterion) بـ (Tong, 1990 ; LeBaron, 1991) (SIC) الذي انثتق بالاعتماد على مفهوم

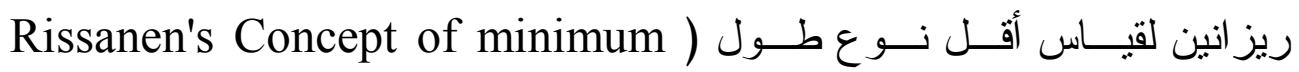
(Mees, 1993) (description length تم بناء نماذج صغيرة بخصائص جيدة وبأقل خطأ ، ويعرف كما يأتي $\operatorname{SIC}(\mathrm{M})=\mathrm{BIC}(\mathrm{M}) / \mathrm{T}$

إذ ان M تمثل عدد معلمات النموذج ، T حجم العينة المستخدمة .

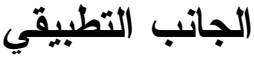

Introduction : المقدمة

في هذا الجانب تم بناء نموذج صغير وذللك بتطبيق طريقة دالـــة القاعـــة النصف قطرية (الثعاعية)(radial basis method) ، وكنلك اختيار حجم النموذج الأمتل باستخدام قو انين الطول(Rissanen, 1989). تم اخذ سلسلة البقع الثمســية ، لتطبيق طريقة دالة القاعدة النصف قطرية (الثعاعية) (radial basis method)

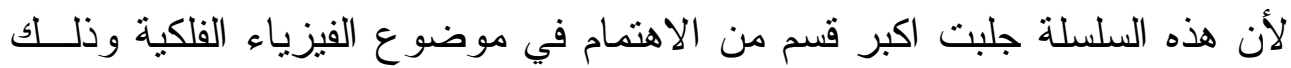

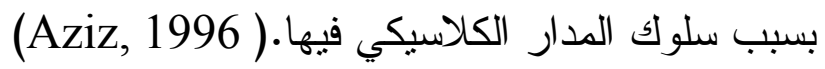




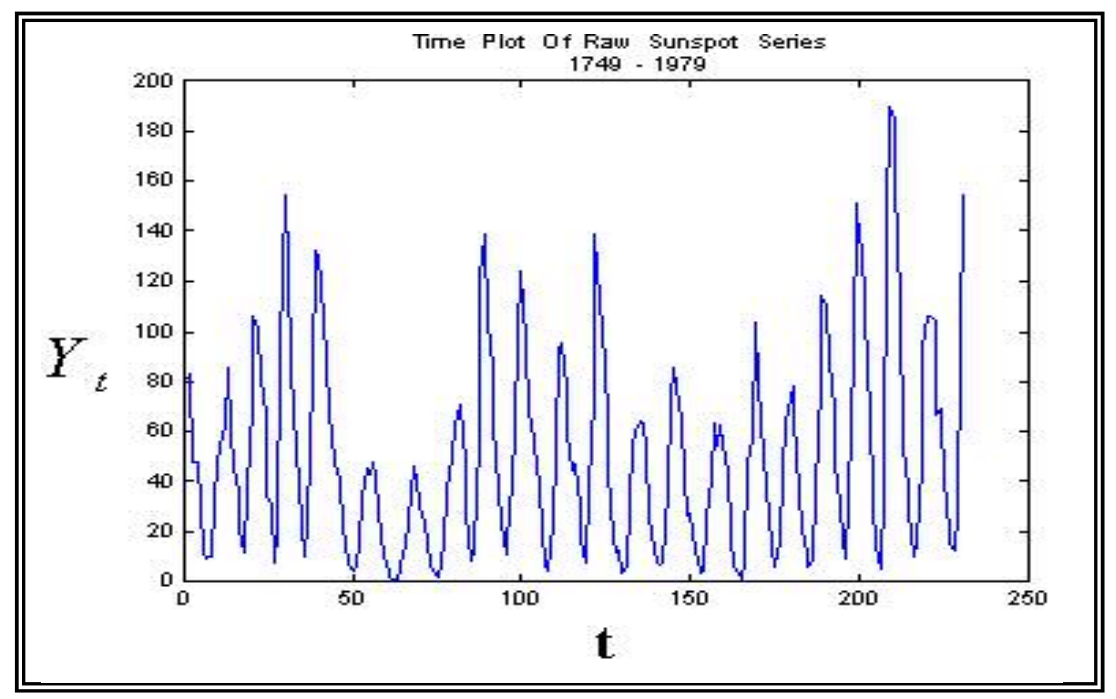

الثشكل (1)

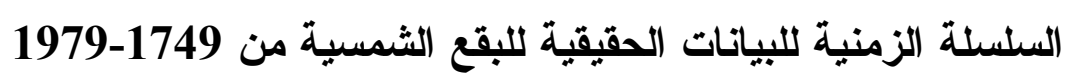

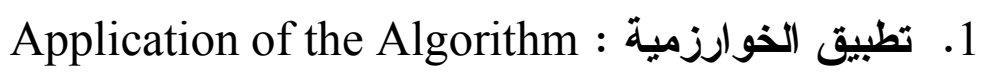

بعد نطبيق نظرية Taken’s الموضحة في فقرة (2) وباستخدام بر امج بلغة

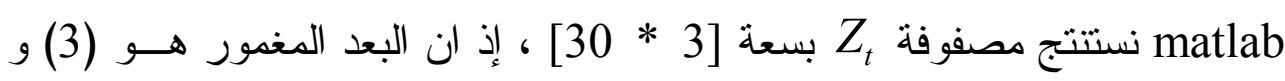

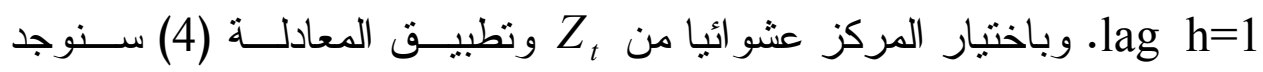

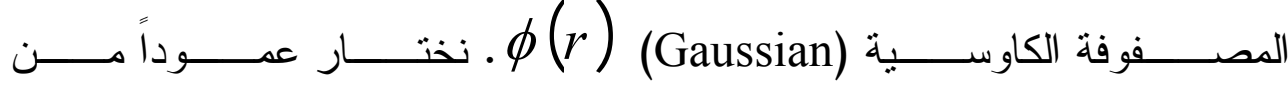

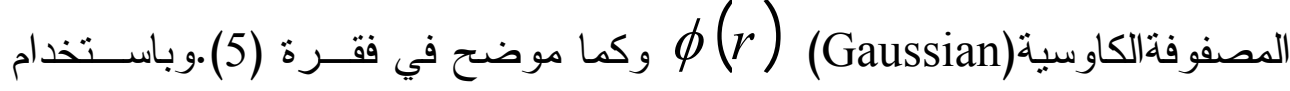
السلسلة الزمنية الحقيقية yt ، ينم تطبيق الخوارزمية الموضحة في فقرة(4) ـ سينتج

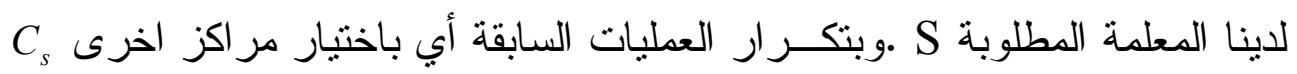

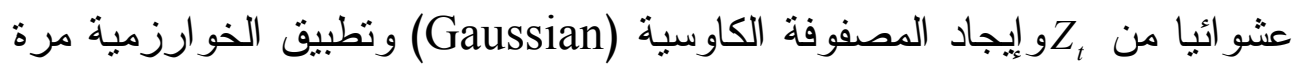

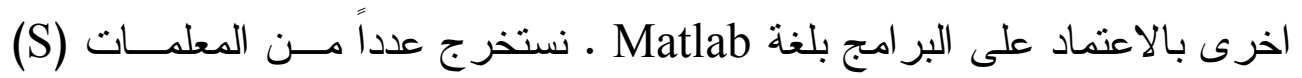
بعدد المر اكز المختارة عشو ائيا .

2. تطبيق نظرية دالة القاعدة النصف قطرية(الثعاعية) بتطبيق المعادلة (8) عند كل معلمة ناتجة في الفقرة (1) وذللك باســتخدام

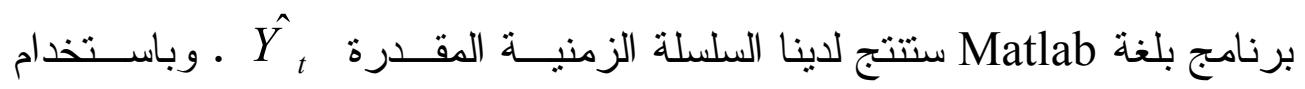

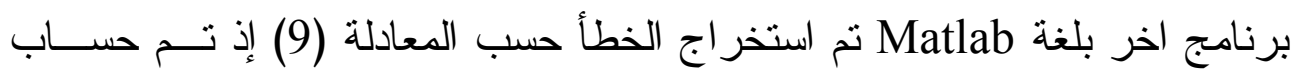




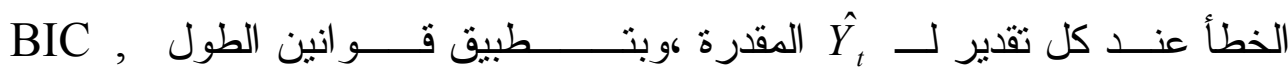
كما في المعادلتين (16) و (15) • وباستخدام برنسامج بلغــــ SIC حساب BIC و SIC عند كل تقدير الى 3. اختيار النموذج الأمثل :

يمكن ملاحظة النموذج الجيد الذي يكون الخطأ فيه قليلاً عندها يتم اختبار

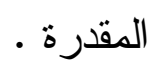

ويمكن ملاحظة ذلك من الثكل (2) إذ ان السلسلة الحقيقية تكون مقاربة من السلسلة

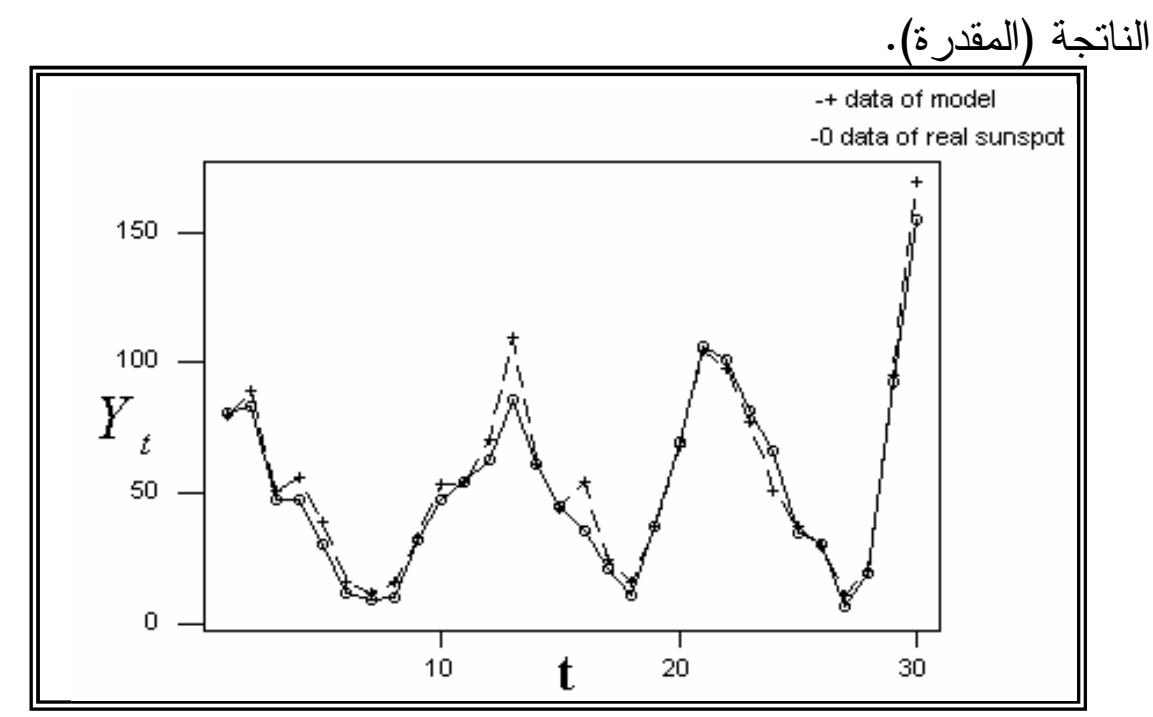

الثنكل (2)

السلسلة الزمنية المقدرة باستخدام نظرية دالة القاعدة (الثُعاعية) والسلسلة الزمنية للبيانات الحقيقية

Covariance : التغاير

ومن خلال البرنامج بلغة Matlab نستخرج التغاير بين عناصر السلسلة

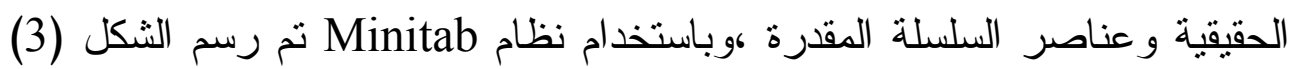

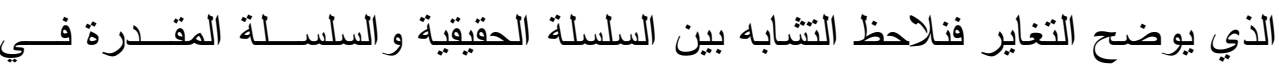

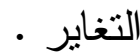




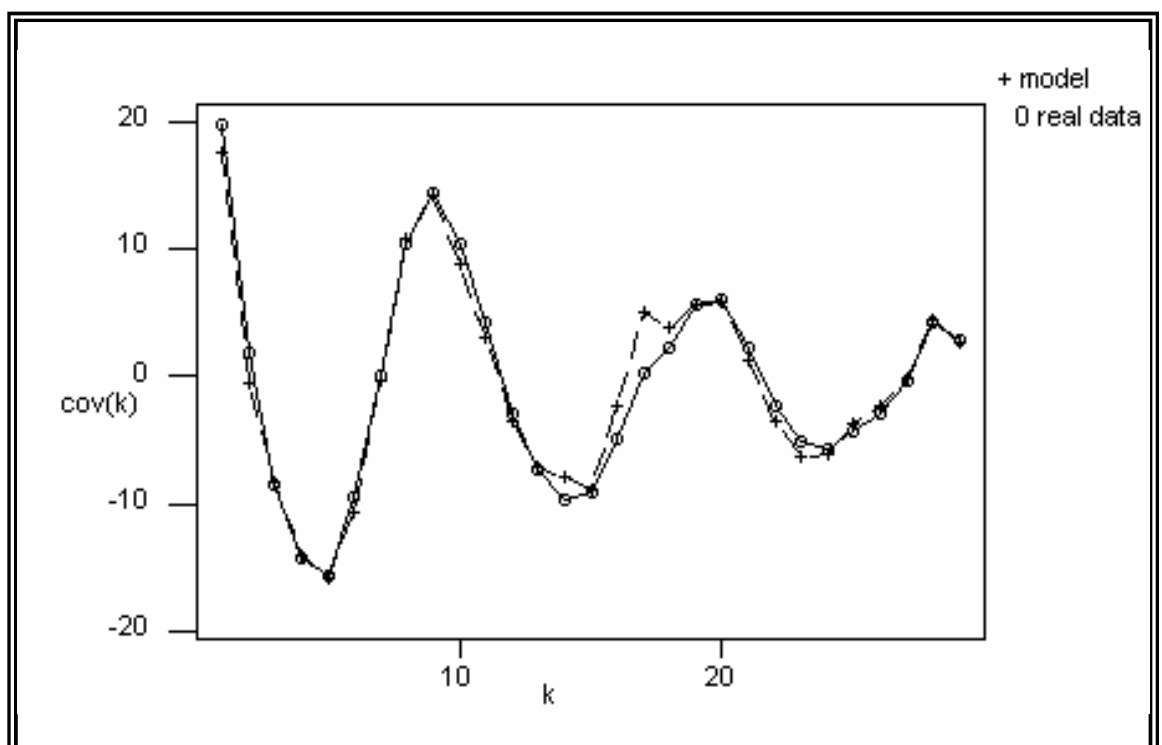

(3) الشكل

التغاير للبيانات الحقيقية وبيانات النموذج المقدرة

كذلك سناحظ أن النموذج الجيد يكون قيمة BIC و SIC عنده اقل ما لإن

بمكن، لاحظ الثكلين (4) و (5) .

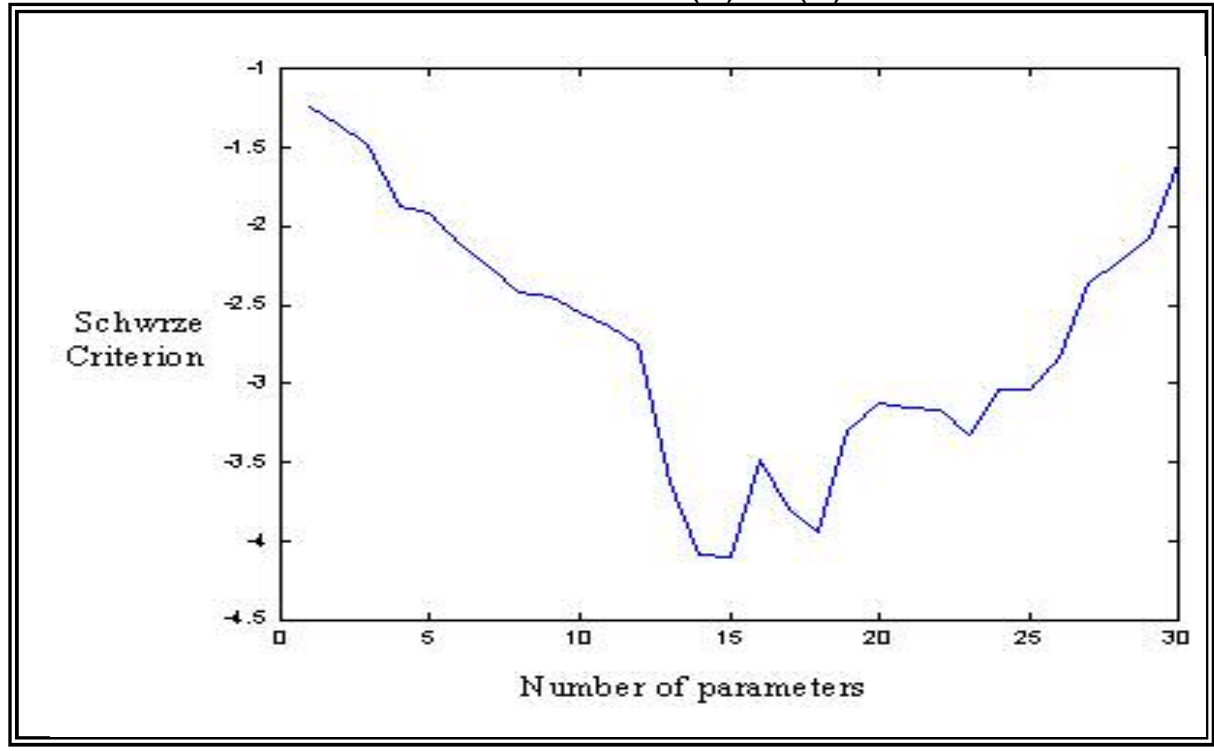

الثكل (4)

مقياس شوارز (Schwrz Information Criterion) بالنسبة الى عدد

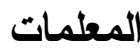




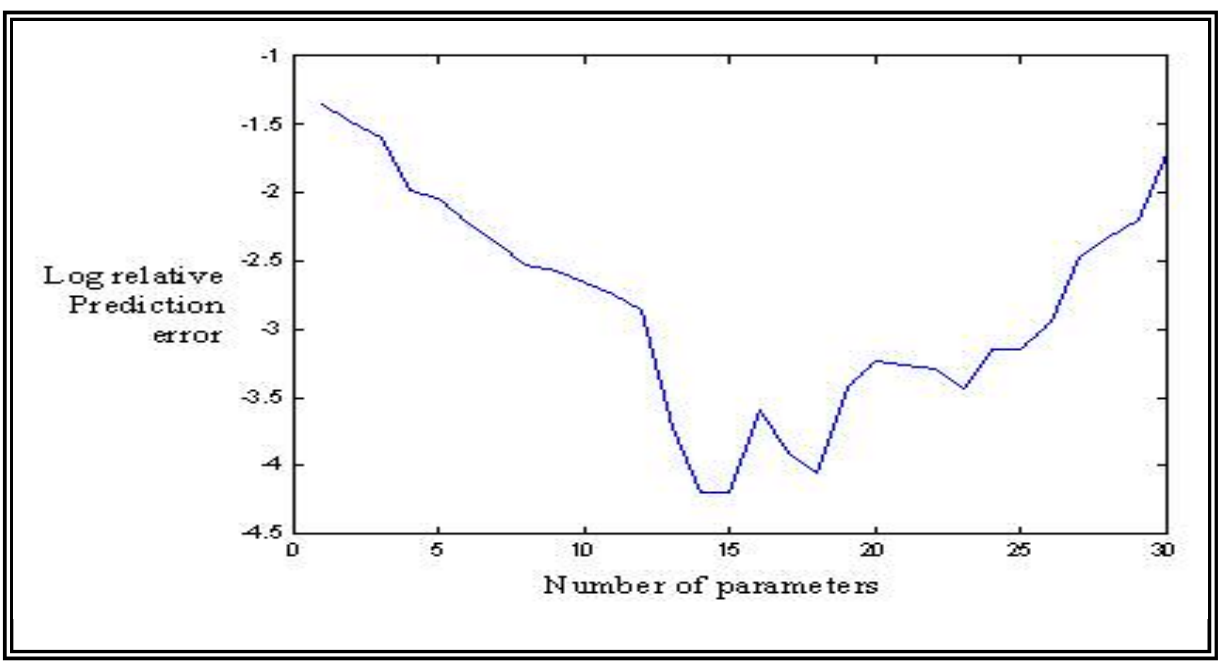

الثكل (5)

Log (err)

5. تحليل البواقي: مesiduals Analysis

بتطبيق المعادلة (1) نحسب البواقي (residuals) كما في الجدول (1) إذ يمثل

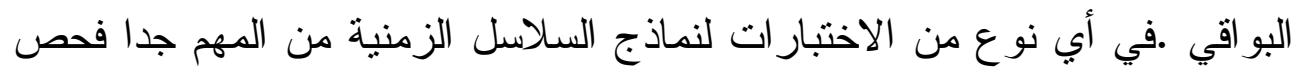

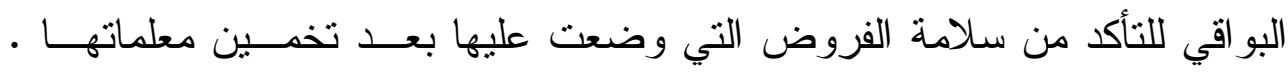

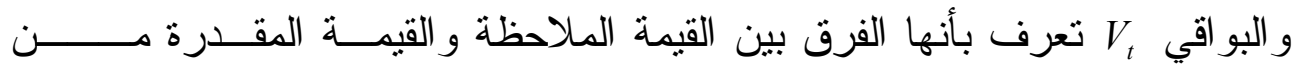

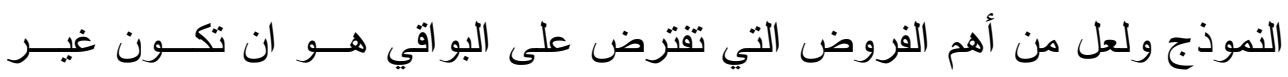

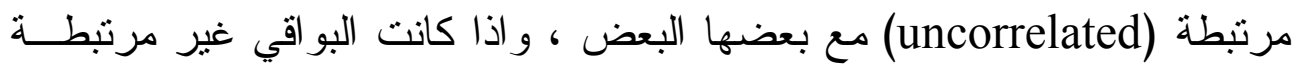

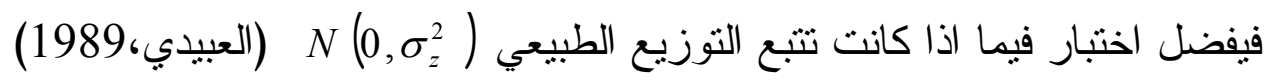

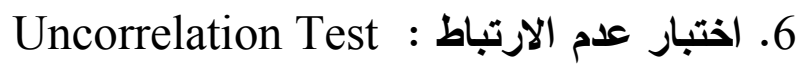

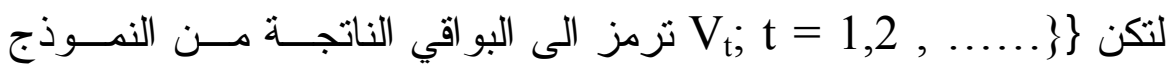
المقتر ح لبيانات السلسلة الزمنيــة \}....

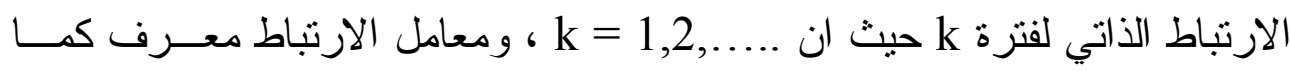

$\left.\left[\hat{r}_{k}=\sum_{t \in k+1}^{n} v_{t}-\bar{v}\right)\left(v_{t-k}-\bar{v}\right) / \sum_{t=1}^{n}\left(v_{t}-\bar{v}\right)^{2}\right]$ يأني : (Aziz, 1996) Where $\quad \bar{v}=n^{-1} \sum_{t=1}^{n} v_{t}$ 


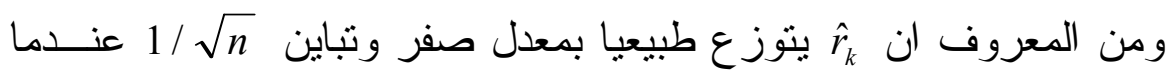

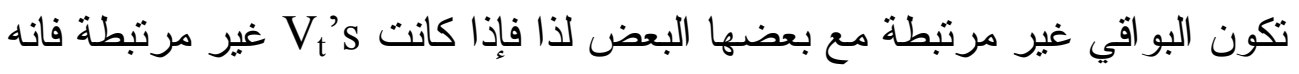

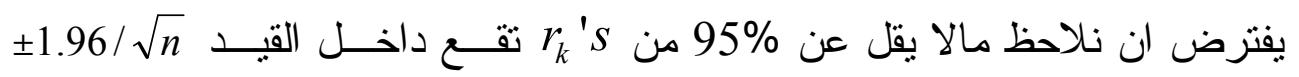

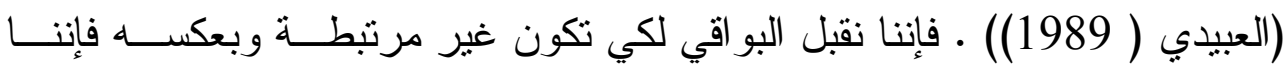

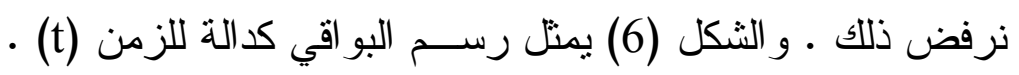
الجدول (1) :(جدول قيم البو اقي)

\begin{tabular}{|c|c|c|c|c|c|c|c|}
\hline $\mathrm{t}$ & $Y_{t}$ & $\hat{Y_{t}}$ & $V_{t}$ & $\mathrm{t}$ & $Y_{t}$ & $\hat{Y_{t}}$ & $V_{t}$ \\
\hline 1 & 80.9 & 80.048 & 0.85 & 16 & 36.0 & 53.998 & -17.9978 \\
\hline 2 & 83.4 & $\mathbf{8 8 . 8 7 7}$ & -5.477 & 17 & 20.9 & 24.591 & -3.6909 \\
\hline 3 & 47.4 & 50.641 & -3.2412 & 18 & 11.4 & 16.345 & -4.9453 \\
\hline 4 & 47.8 & 55.895 & -8.095 & 19 & 37.8 & 37.215 & 0.585 \\
\hline 5 & 30.7 & 39.039 & -8.339 & 20 & 69.8 & 67.643 & 2.1568 \\
\hline 6 & 12.2 & 16.345 & -4.145 & 21 & 106.1 & 104.347 & 1.7529 \\
\hline 7 & 9.6 & 11.529 & -1.929 & 22 & 100.8 & 98.072 & 2.7283 \\
\hline 8 & 10.2 & 15.835 & -5.6345 & 23 & 81.6 & 77.056 & 4.5437 \\
\hline 9 & 32.4 & 33.493 & -1.0932 & 24 & 66.5 & 51.298 & 15.2021 \\
\hline 10 & 47.6 & 53.633 & -6.033 & 25 & 34.8 & 37.215 & -2.4147 \\
\hline 11 & 54.0 & 54.071 & -0.0708 & 26 & 30.6 & 30.064 & 0.5364 \\
\hline 12 & 62.9 & 70.197 & -7.297 & 27 & 7.0 & 11.164 & -4.1644 \\
\hline 13 & 85.9 & 109.674 & -23.7739 & 28 & 19.8 & 20.213 & -0.4127 \\
\hline 14 & 61.2 & 61.368 & -0.1678 & 29 & 92.5 & 95.007 & -2.5069 \\
\hline 15 & 45.1 & 44.001 & 1.0991 & 30 & 154.4 & 169.290 & -14.8904 \\
\hline
\end{tabular}




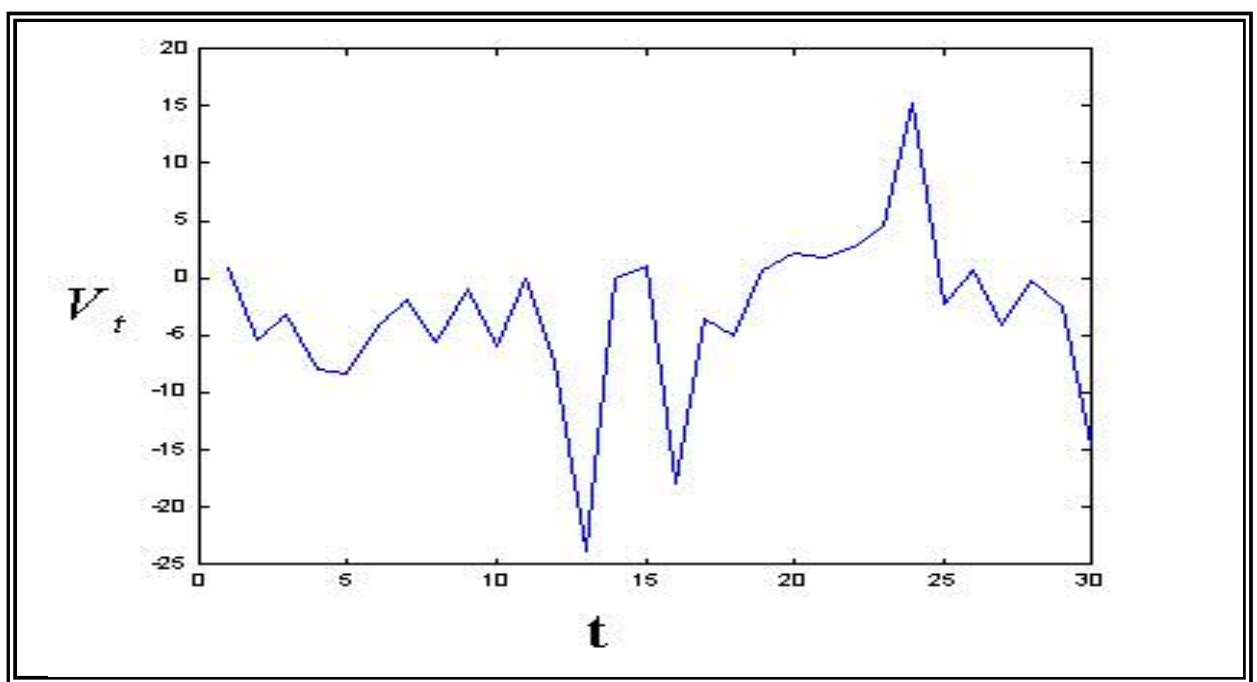

(6) الثكل

البواقي كدالة للزمن بالنسبة الى نموذج دالة القاعدة النصف قطرية (الثعاعية)

7est of Normality : اختبار الطبيعية

وباستخدام نظام Minitab تم حساب دالة الارتباط الذاتي للبو اقي وبحيث

ان دالة الارتباط الذاتي هنا هي 30,..,

99\%

ملاحظة الثكل (7) نلاحظ ان دالة الارتباط الذاتي تبقى داخل القيد في الرسم وهذا

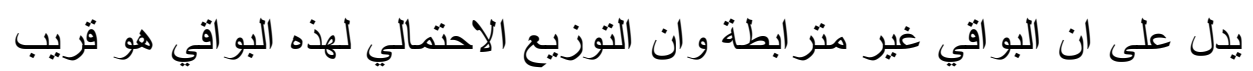

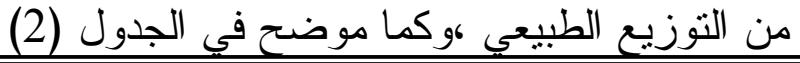

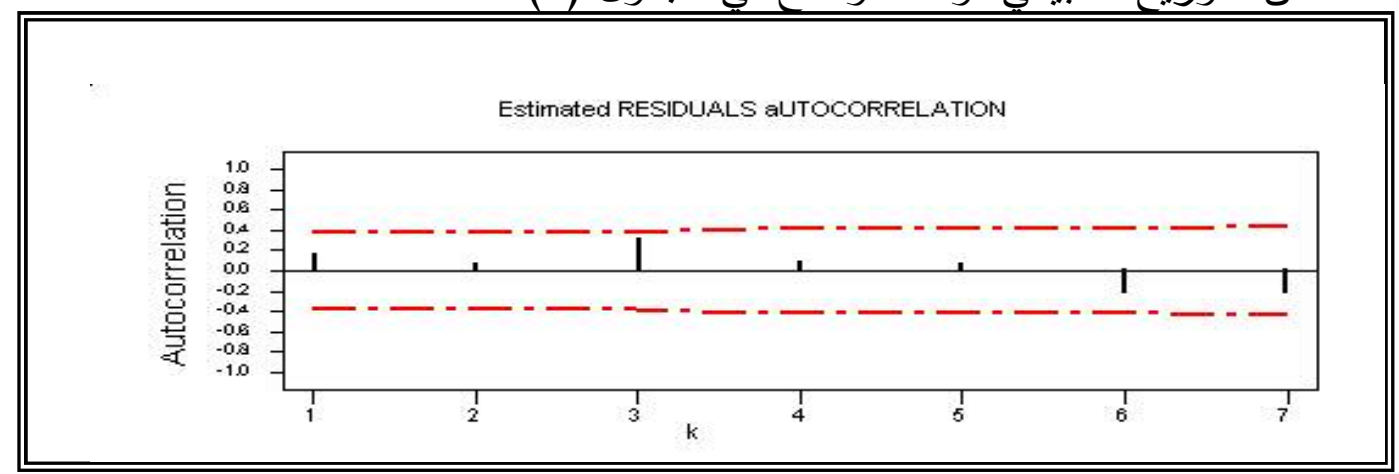

الثكل (7) :الارتباط الذاتي لبو اقي نموذج دالة القاعدة النصف قطرية (الشعاعية) الجدول (2) :جدول اختبار البواقي لمودج دله 


\begin{tabular}{|c|c|c|c|c|}
\hline الحد الأدنى & الحد الأعلى & المشاهدة & المتوقيم & Chi-square \\
\hline At or below & -23 & 1 & 0.105 & 7.63 \\
\hline-23 & -20 & $\mathbf{0}$ & 0.216 & 0.216 \\
\hline-20 & -17 & 1 & 0.54 & 0.392 \\
\hline-17 & -14 & 1 & 1.344 & 0.09 \\
\hline-14 & -11 & $\mathbf{0}$ & 2.271 & 2.27 \\
\hline-11 & -8 & 2 & 3.453 & 0.61 \\
\hline-8 & -5 & 4 & 4.458 & 0.047 \\
\hline-5 & -2 & 7 & 4.992 & 0.081 \\
\hline-2 & 1 & 8 & 4.392 & 2.964 \\
\hline 1 & 4 & 4 & 3.891 & 0.003 \\
\hline 4 & 7 & 1 & 2.046 & 0.535 \\
\hline 7 & 10 & $\mathbf{0}$ & 1.305 & 1.305 \\
\hline 10 & 13 & 0 & 0.621 & 0.621 \\
\hline 13 & 16 & 1 & 0.261 & 2.09 \\
\hline Total & & 30 & & 18.853 \\
\hline \multicolumn{4}{|c|}{$\chi_{11.0 .01}^{2}=\mathbf{2 4 . 7 2}$} & Accept $\mathbf{H}_{0}^{*}$ \\
\hline \multicolumn{4}{|c|}{$\chi_{11.0 .05}^{2}=\mathbf{1 9 . 6 8}$} & $\overline{\text { Accept } \mathrm{H}_{0}}$ \\
\hline
\end{tabular}

$\mathrm{H}_{0}^{*}$ : The residuals are normally distributed

نلاحظ ان القيمة المحسوبة في الجدول هي ( 18.853 ) أقل من القيمة الجدولية

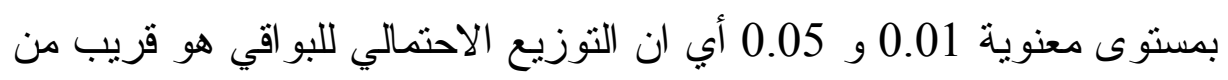

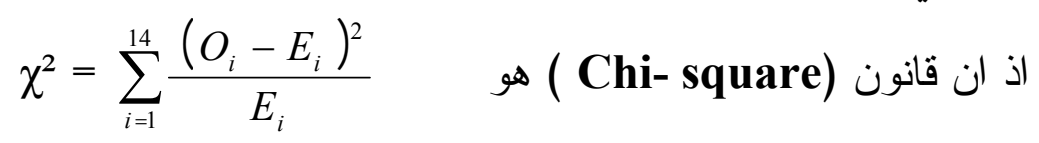

حيث ان E $E_{i}$

(9) يمكن ملاحظة الثكل (8) للاحتمال الطبيعي للبو اقي وكذلك الثنكل

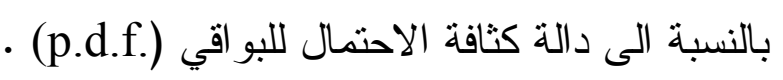



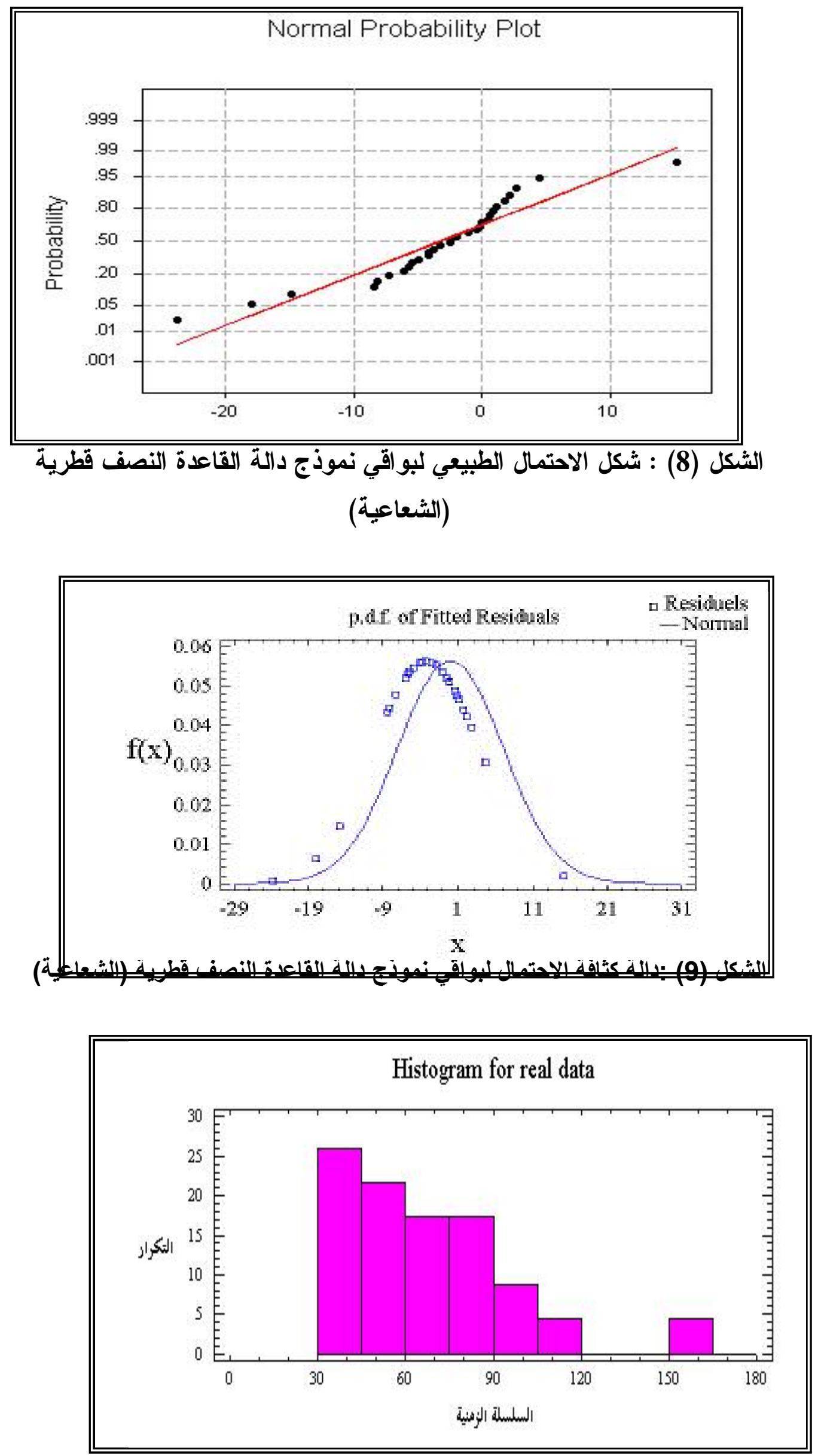
الثكل (10): المدرج التكراري للبيانات الحقيقية

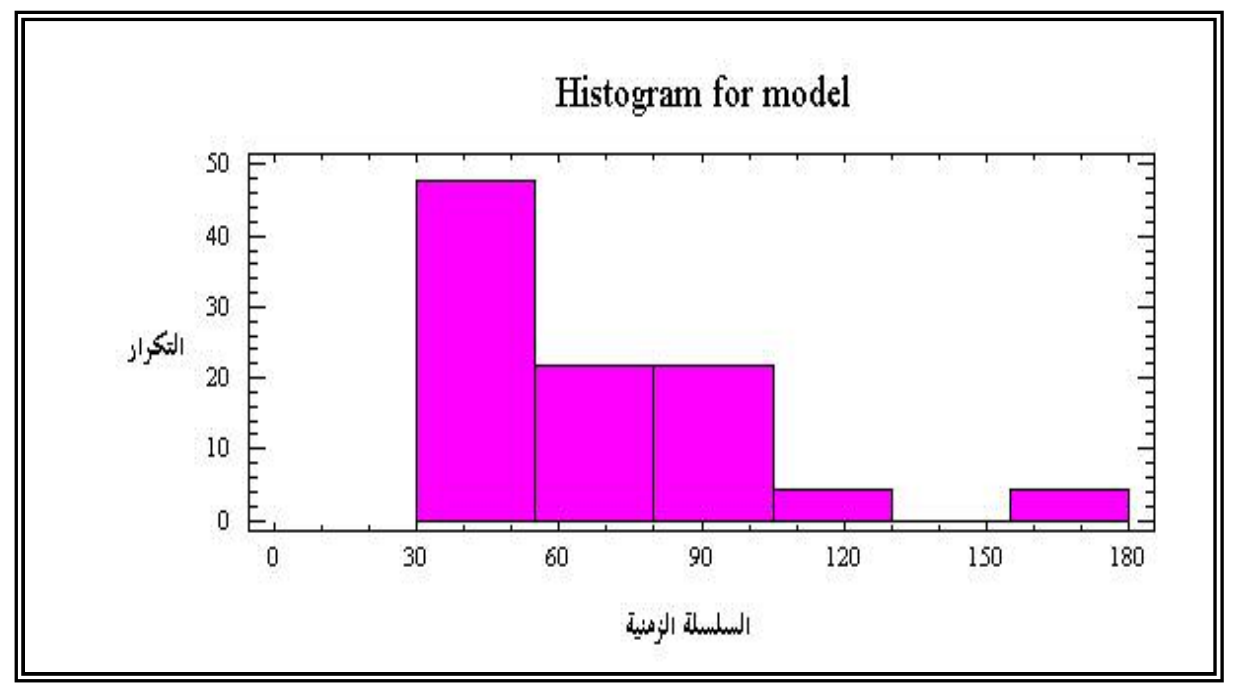

الشكل (11) :المدرج التكراري لبيانات النموذج الشعاعي

تبين من الثكلين (10) و (11) أنهما منطابقان من حيث الثباه بثكل الدالة

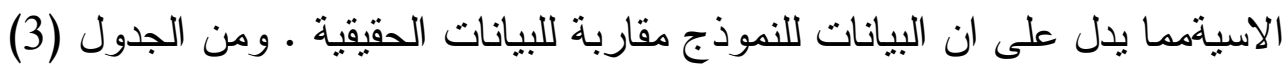

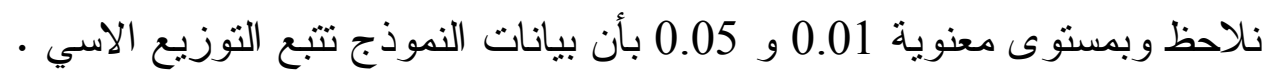

(3) (الجدول

\begin{tabular}{|c|c|c|c|c|}
\hline الد الأدنى & الحد الأعلى & المشاهدة & القيم المتوقعة & Chi- square \\
\hline 0 & 13 & 2 & 6.15 & 2.8 \\
\hline
\end{tabular}




\begin{tabular}{|c|c|c|c|c|}
\hline 13 & 26 & 5 & 4.89 & 0.0025 \\
\hline 26 & 39 & 4 & 3.867 & 0.0046 \\
\hline 39 & 52 & 3 & 3.093 & 0.0028 \\
\hline 52 & 65 & 6 & 2.46 & 5.09 \\
\hline 65 & 78 & 3 & 1.95 & 0.565 \\
\hline 78 & 91 & 2 & 1.55 & 0.131 \\
\hline 91 & 104 & 2 & 1.23897 & 0.467 \\
\hline 104 & 117 & 2 & 0.99 & 1.030 \\
\hline 117 & 130 & $\mathbf{0}$ & 0.771 & 0.771 \\
\hline 130 & 143 & 0 & 0.62265 & 0.623 \\
\hline 143 & 156 & $\mathbf{0}$ & 0.49455 & 0.494 \\
\hline 156 & 169 & $\mathbf{0}$ & 0.3933 & 0.3933 \\
\hline 169 & 182 & 1 & 0.3135 & 1.5033 \\
\hline Total & & 30 & & 13.8775 \\
\hline \multicolumn{4}{|c|}{$\chi_{12.0 .01}^{\mathbf{2}}=\mathbf{2 6 . 2 1 7}$} & Accept $\mathbf{H}_{0}^{*}$ \\
\hline \multicolumn{4}{|c|}{$\chi_{12.0 .05}^{2}=21.0261$} & Accept $\mathrm{H}_{0}$ \\
\hline
\end{tabular}

$\mathrm{H}_{0}^{*}$ : the data of model are exponential distribution

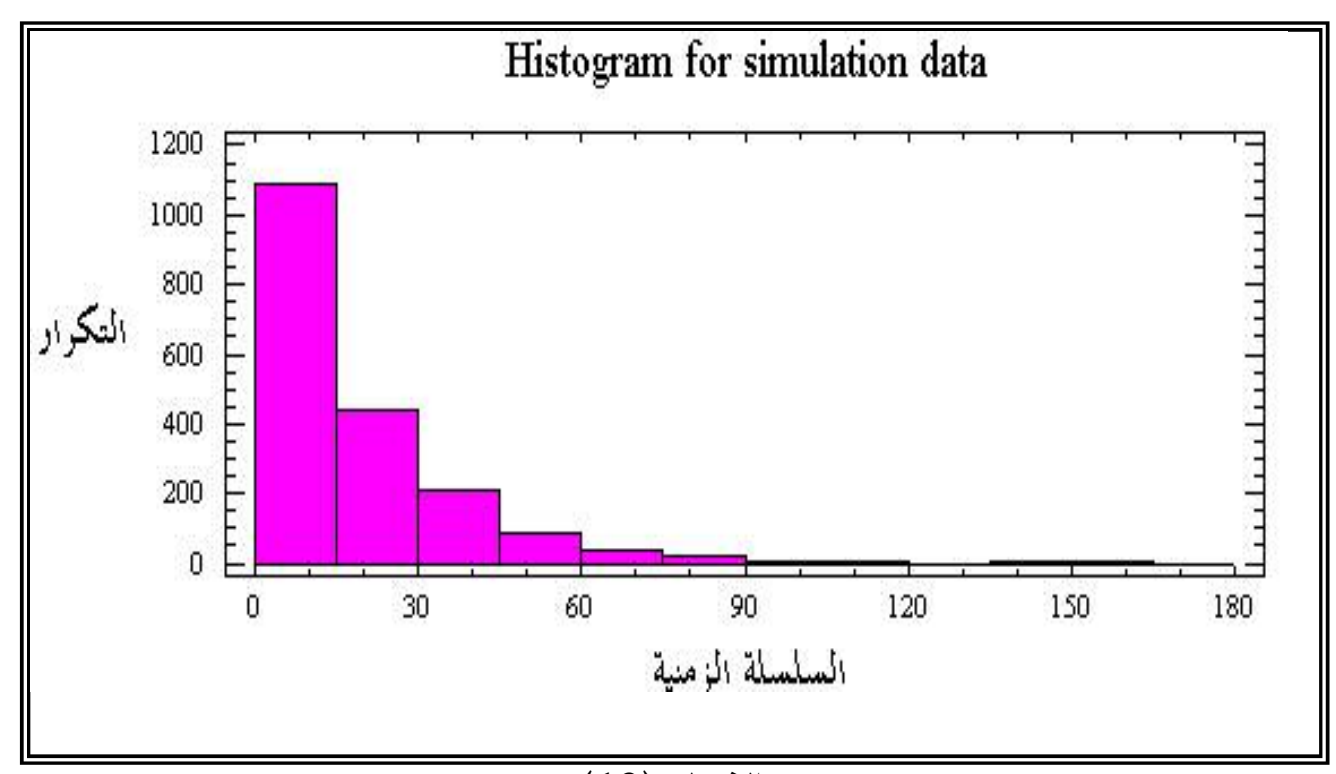

الثُكل (12)

المدرج التكراري للبيانات المولدة من نموذج الثعاعي 
8imulation of Exponential : المحاكاة باستخدام الثبه بالدالة الاسية Function من رسم الثكل (12) يمكن ملاحظة أن بيانات النموذج تتبع التوزيع الاسي

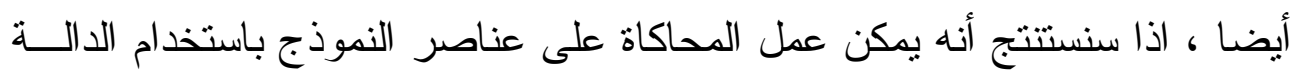
الاسية ـ ومن خلال نظام Minitab تم توليد بيانات من الدالة الاسية لعمل المحاكاة

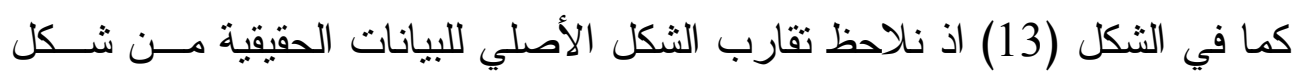

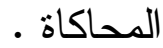
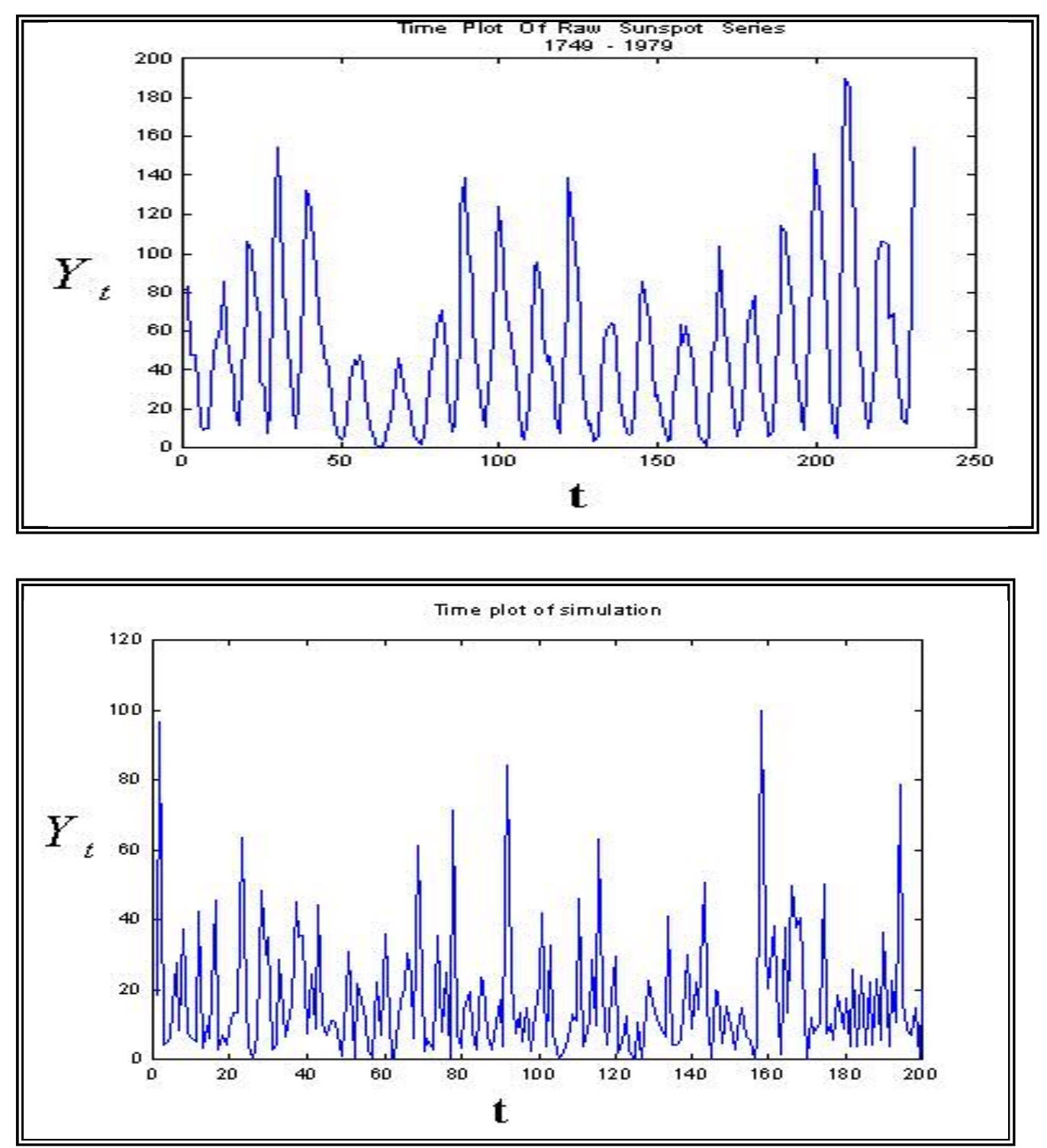


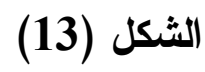

مقارنة بين بيانات السلسلة الزمنية الحقيقية للبقع الثمسية وبيانات المحاكاة لنموذج دالة القاعدة الثعاعية

Conclusion: الاستنتاجات

نستتتج من تطبيق طريقة دالــة القاعــدة النصــف قطريـــة (الثـــعاعية)

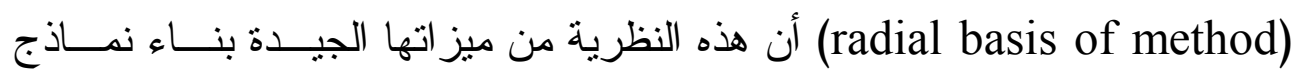

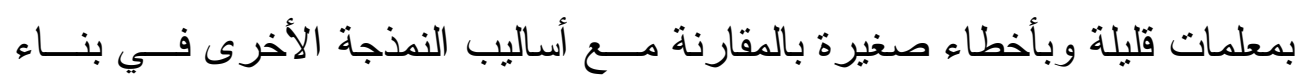

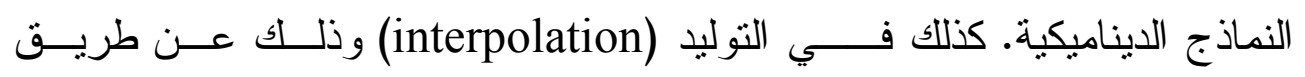

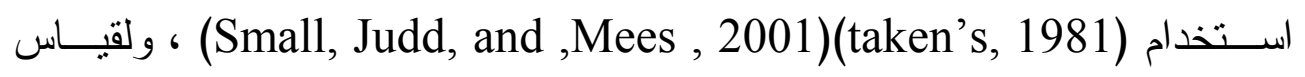

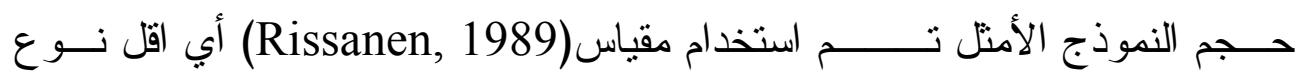

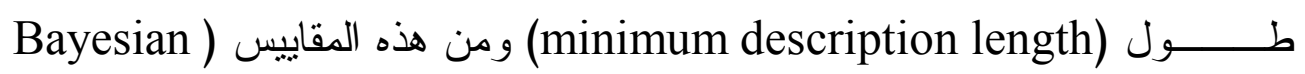
Schwarz و ويرمسـز لـــ للاختصــار (Information criterion (information criterion

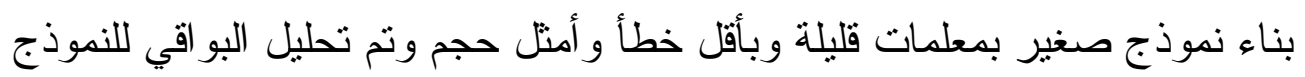
بالاعتماد على اختبار عدم الارتباط (Uncorrelation Test) و واختبار الطبيعيــة ولئة وكذللك تم عمل المحاكاة لبيانات النموذج.

Recommendation : التوصيات

1. بناء نماذج لسلاسل زمنية مختلفة تؤخذ من الطبيعة باســتعمال طريقــة دالـــة

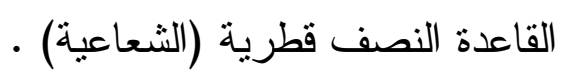


2. بناء نموذج لسلاسل زمنية باستعمال طريقة القاعدة النصف قطرية (الثعاعية)

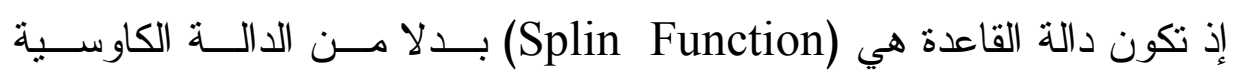
التي تم استعمالها في هذا البحث. (Gaussian Function)

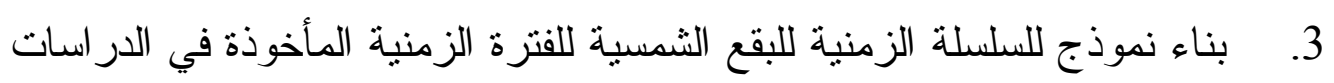

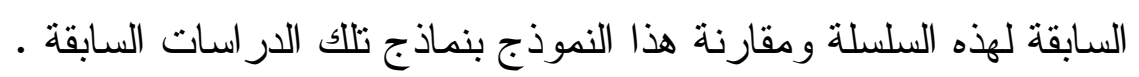

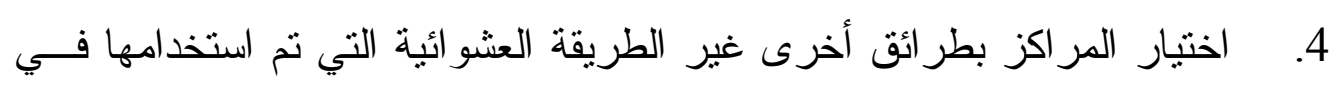
هذا البحث.

5. استعمال النظام الديناميكي المولد بطريقة دالة القاعدة النصف قطرية (الثعاعية)

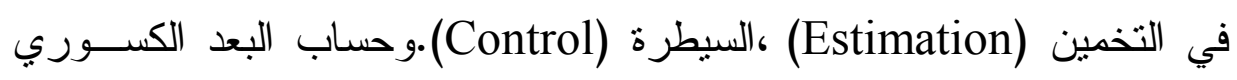
الاحتمالي.

$$
\text { - المصادر }
$$

1. العبيدي ، عبد الغفور جاسم (1989) ، "تحليل ونمذجــة السلســلة الزمنيـة

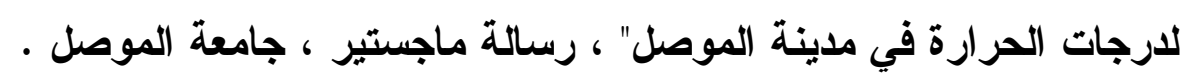

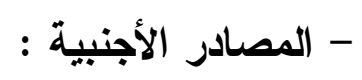

2. Aziz, M.M. (1996), "A New Analysis of Sunspot time Series”, Ph.D. thesis, University of Mosul.

3. Casdagli, M. (1989), "Non - Linear Prediction of Chaotic Time Series", Physica D35(3), 335 - 356.

4. Farmer, J.D. and Sidorowich, J.J. (1987), "Predicting Chaotic Time Series", Phys. Rev. Lett., 59(8), 845 - 848.

5. Grassberger, P. and Procaccia, I. (1983), "Characterization of Strange Ahractor", Physics Review Letter So: 346.

6. Judd, K. and Mees, A. (1995), "On Selecting Models for Non Linear Time Series”, Physica D82: 426 - 444. 
7. Mees, A.I., (1990), "Modeling Complex Systems", In: Dynamics of Complex Interconnected Biological Systems eds. Vincent, T., Mees, A.I. and Jennings, L.S., Birkhauser, Boston, pp. $104-124$.

8. Mees, A.I., (1993), "Parsimonious Dynamical Reconstruction", The University of Western Australia, In: Bifurcation and Chaos3, 669-675.

9. Mees, A.I., (1994), "Reconstruction Chaotic Systems in the Presence of Noise", The University of Western Australia, Nedlands 6009, pp. $305-321$.

10. Rissanen, J. (1989), "Stochastic Complexity in Statistical Inquiry", World Scientific Singapore.

11. Small, M. and Judd, K. and Mees, A. (2001), "Testing Time Series for Nonlinearly", University of Western Australia. Statistics and Computing, 11: $257-268$.

12. Small, M., Judd, K. and Mees, A. (2002), "Modeling Continuous Process Form Data”, Physical Review, volume E65, 046704.

13. Takens, F. (1981), "Detecting Strange Attractors in Turbulence", Lecture Notes in Mathematics 898: 366 - 381.

14. Tong, H. (1990), "Non-linear Time Series: A Dynamical System", Approach Oxford Clarendon.

15. William W.S. Wel (1989), "Time Series Analysis", Department of Statistics Temple University. 